\title{
Probiotics Mediated Modulation of Gut-Flora Might Be a Biotherapeutical Approach for Obesity and Type 2 Diabetes
}

\author{
Hariom Yadav ${ }^{1 *}$, Shalini Jain ${ }^{2}$ and Francesco Marotta ${ }^{3 *}$
}

${ }^{1}$ Diabetes, Endocrinology and Obesity Branch, National Institute of Diabetes, Digestive and Kidney Diseases, National Institutes of Health, Bethesda, MD, USA ${ }^{2}$ Laboratory of Bio-Organic Chemistry, National Institute of Diabetes, Digestive and Kidney Diseases, National Institutes of Health, Bethesda, MD, USA

${ }^{3}$ ReGenera Research Group for Aging Intervention, Milan, Italy

Increasing prevalence of obesity and type 2 diabetes (T2D) Figure 1 around the globe, creating higher risk for cardiovascular diseases (CVDs) and increasing strain on health care budget of every country in the world [1]. This is because of unavailability of successful preventive/ therapeutic strategies against these health ailments. The reason behind this problem is the complex pathophysiology of obesity and T2D [2]. Various factors i.e. genetics, environmental factors and diet plays an important role in development of obesity and progression of its life threatening complications i.e. insulin resistance (T2D) and CVDs, through modulation of various fundamental metabolic pathways i.e. food intake, lipid metabolism (hyperlipidemia), oxidative stress and immune system (inflammation) [3]. Recently, role of gut-flora in development of obesity and T2D got tremendous attention among scientific community and has been considered one the potential therapeutical targets against obesity and diabetes [4-6]. Probiotics are potential modulators of gut-flora that changes gut-flora composition in a beneficial manner and exerts various health beneficial effects i.e. anti-hyperlipidemic, anti-oxidant and anti-inflammatory [7]. But the consumption of probiotics for human is cautioned and highly debatable in terms of development of obesity and weight gain. Here we will discuss few points and scientific findings/ reasons about how probiotics can be used as a biotherapeutical agent against obesity and diabetes.

This concerned arisen for probiotic consumption in the report of Dr. Raoult [8] that was on the basis of animal data, where sick animals treated with probiotics start gaining weight rapidly after probiotic treatment $[9,10]$. But there are no evidences suggest that animals treated with probiotics became obese/ diabetic. Our previous studies suggested that feeding of probiotic supplemented milk product (dahi/ yogurt) suppressed progression of diet induced T2D Figure 1 and metabolic derangements $[11,12]$. In addition, our ongoing study also suggested (un-published data) that consumption of probiotic formulations dramatically suppressed obesity and diabetes in high fat diet fed mice and reduced food intake. This suggests that, consumption of probiotics is not deleterious for progression of obesity and T2D. However,
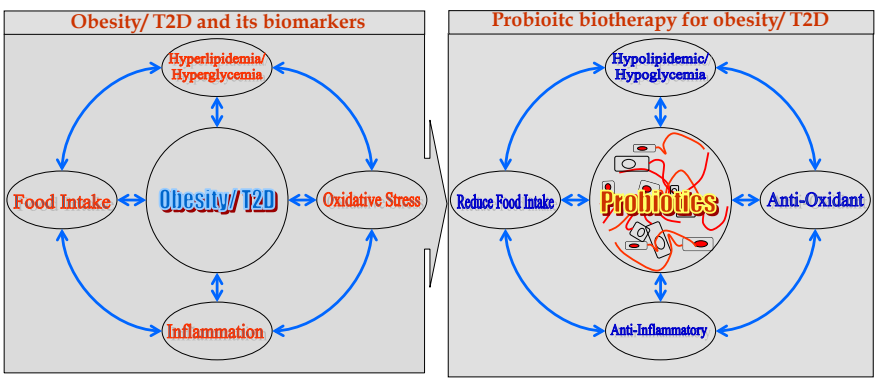

Figure 1: Obesity/ type 2 diabetes (T2D) and their biomarkers i.e.hyperlipidemia / hyperglycemia, oxidative stress, inflammation and food intake (left) and how probiotics might act to ameliorate the risk of obesity / T2D by targeting these biomarkers (right). these evidences doesn't exclude the differential efficacy of probiotics in human subjects, hence very well controlled/ designed studies are urgently needed to check the efficacy of probiotics in human health against obesity and diabetes. A deeper knowledge of our gut ecology will be mandatory in the hope to target much more specific probiotic intervention. In this regards it is of interest the recent intriguing review from Rastmanesh [13] suggesting that a high-polyphenol and selectively-restricted probiotic diet could represent a novel strategy for planning a successful dietary regimen and/or neutraceutical/ pharmaceutical preparations to achieve and maintain a normal body weight in obese individuals. Overall, such studies will also give opportunities to industries to think about the risk vs safety of probiotic formulations for obesity and diabetes in consumers, worldwide.

\section{References}

1. Hossain P, Kawar B, Nahas ME (2007) Obesity and Diabetes in the Developing World-A Growing Challenge. N Engl J Med 356: 213-215

2. Lean ME (2000) Pathophysiology of obesity. Proc Nutr Soc 59: 331-336.

3. Lopez RP (2007) Neighborhood Risk Factors for Obesity. Obesity 15: 2111 2119

4. Bajzer M, Seeley RJ (2006) Physiology: Obesity and gut flora. Nature 444 1009-1010.

5. Ley RE, Turnbaugh PJ, Klein S, Gordon JI (2006) Micro Ecol: human gut microbes associated with obesity. Nature 444: 1022-1023.

6. Turnbaugh PJ, Ley RE, Mahowald MA, Magrini V, Mardis ER, et al. (2006) An obesity-associated gut microbiome with increased capacity for energy harvest Nature 444: 1027-1031.

7. Yadav H, Jain S, Sinha PR, Marrota F (2007) Diabetes and probiotics: A possible therapeutic link. Int J Probiotics Prebiotics 2: 15-20.

8. Raoult D (2009) Probiotics and obesity: a link? Nat Rev Microbiol 7: 616.

9. Ehrlich SD (2009) Probiotics - little evidence for a link to obesity. Nat Rev Microbiol 7: 901

10. Delzenne N, Reid G (2009) No causal link between obesity and probiotics. Nat Rev Microbiol 7: 901

*Corresponding authors: Hariom Yadav, Diabetes, Endocrinology and Obesity Branch, National Institute of Diabetes, Digestive and Kidney Diseases, National Institutes of Health, Bethesda, MD, USA, E-mail: yadavhariom@gmail.com

Francesco Marotta, ReGenera Research Group for Aging Intervention, Texas University, Department of Human Nutrition, Piazza Firenze, Milano, Italy, Tel: 39024077243; E-mail: fmarchimede@libero.it

Received August 25, 2011; Accepted November 16, 2011; Published November 18, 2011

Citation: Yadav H, Jain S, Marotta F (2011) Probiotics Mediated Modulation of Gut-Flora Might Be a Biotherapeutical Approach for Obesity and Type 2 Diabetes. Metabolomics 1:107e. doi:10.4172/2153-0769.1000107e

Copyright: (C) 2011 Yadav H, et al. This is an open-access article distributed unde the terms of the Creative Commons Attribution License, which permits unrestricted use, distribution, and reproduction in any medium, provided the original author and source are credited. 
Citation: Yadav H, Jain S, Marotta F (2011) Probiotics Mediated Modulation of Gut-Flora Might Be a Biotherapeutical Approach for Obesity and Type 2 Diabetes. Metabolomics 1:107e. doi:10.4172/2153-0769.1000107e

Page 2 of 2

11. Yadav H, Jain S, Sinha PR (2007) Antidiabetic effect of probiotic dahi containing Lactobacillus acidophilus, Lactobacillus casei and Lactococcus lactis bacteria in high fructose diet fed rats. Nutrition 23: 62-68.

12. Yadav H, Jain S, Sinha PR (2008) Oral administration of dahi containing probiotic Lactobacillus acidophilus and Lactobacillus casei ameliorated the Streptozotocin-induced oxidative stress and dyslipidemia in rats. J Dairy Res 75: 189-195.

13. Rastmanesh $R$ (2011) High polyphenol, low probiotic diet for weight loss because of intestinal microbiota interaction. Chem-Bio Interact 189: 1-8. 